\title{
NeuroRegulation
}

\section{Editorial - Volume 6, Number 2}

Citation: Cannon, R. L. (2019). Editorial - Volume 6, Number 2. NeuroRegulation, 6(2), 2. https://doi.org/10.15540/nr.6.2.53

Copyright: (c) 2019. Cannon. This is an Open Access article distributed under the terms of the Creative Commons Attribution License (CC-BY).
*Address correspondence to: Rex L. Cannon, PhD, BCN, Knoxville Neurofeedback Group, 7147 Kingston Pike, Ste 103, Knoxville, TN 37919, USA. Email: rcannonphd@gmail.com
Welcome to NeuroRegulation 6, Issue 2; thanks for joining us for the latest issue. In the current issue authors utilize a variety of research techniques and several case reports demonstrating interesting findings. Ashlie N. Bell, Donald Moss, and Robert J. Kallmeyer present data of a controlled study examining LORETA Z-Score neurofeedback and HRV biofeedback for chronic posttraumatic stress disorder (PTSD). Michela Balconi and Maria Elide Vanutelli present data for optical imaging and neurofeedback intervention for emotional behavior regulation in schizophrenia. Jon A. Frederick, Andrew S. Heim, and Kelli N. Dunn present data concerning self-prompted discrimination and operant control of the alpha frequency domain. Rubén PérezElvira, José A. Carrobles, Diego J. López Bote, and Javier Oltra-Cucarella present case study data for the efficacy of Live Z-score neurofeedback training in chronic insomnia. And finally, George Lindenfeld, George Rozelle, John Hummer, Michael R. Sutherland, and James C. Miller present a case study of remediation of PTSD in a combat veteran.

NeuroRegulation thanks these authors for their valuable contributions to the scientific literature for neurofeedback, neuroscience, and learning. We strive for high quality and interesting empirical topics. We encourage the members of ISNR and other biofeedback and neuroscience disciplines to consider publishing with us. We extend an invitation to all researchers and clinicians interested in human performance, the human brain, and methods to improve its functionality to submit reviews, theoretical articles, and research data. It is important to stress that publication of case reports is also always useful in furthering the advancement of an intervention for both clinical and normative functioning. We encourage researchers, clinicians, and students practicing neurofeedback to submit case studies, or groups of case studies!

NeuroRegulation has made great strides for increasing the scientific integrity of neurofeedback, biofeedback, and applied neuroscience. We would like to thank our editorial board, reviewers, and contributors for this success. When writing this editorial, I decided to conduct a search of PubMed with the term "neurofeedback" dated from 1995 to current and there is a substantial increase in the number of articles over the last few years, and we expect this trend to continue. If we are clear to purpose, consistent with methods and publishing outcomes, then we are capable of much. I look forward to more discoveries and processes uncovered to aid in improving human performance across all functional domains.

We thank you for reading NeuroRegulation!

Rex L. Cannon, PhD, BCN

Editor-in-Chief

Email: rcannonphd@gmail.com

Published: June 26, 2019 